Communication et organisation

42 | 2012

La communication, dimension oubliée de l'intelligence économique

\title{
La perception de la confiance informationnelle
}

Impacts sur les comportements informationnels et les pratiques documentaires en contexte organisationnel

Dominique Maurel et Aïda Chebbi

\section{OpenEdition}

\section{Journals}

Édition électronique

URL : http://journals.openedition.org/communicationorganisation/3864

DOI : 10.4000/communicationorganisation.3864

ISSN : 1775-3546

\section{Éditeur}

Presses universitaires de Bordeaux

Édition imprimée

Date de publication : 1 décembre 2012

Pagination : 73-90

ISBN : 978-2-86781-772-4

ISSN : 1168-5549

\section{Référence électronique}

Dominique Maurel et Aïda Chebbi, «La perception de la confiance informationnelle », Communication et organisation [En ligne], 42 | 2012, mis en ligne le 01 décembre 2014, consulté le 10 décembre 2020 URL : http://journals.openedition.org/communicationorganisation/3864 ; DOI : https://doi.org/ 10.4000/communicationorganisation.3864 


\title{
La perception de la confiance informationnelle : impacts sur les comportements informationnels et les pratiques documentaires en contexte organisationnel
}

\author{
Dominique Maurel', Aïda Chebbi²
}

\section{Introduction et problématique}

La confiance est reconnue comme étant à la base de toute relation de coopération. Dans les organisations, si la confiance favorise le partage d'information, l'information serait alors considérée comme un stimulateur de confiance (Huotari \& Iivonen, 2004 ; Vallée \& Mackaay, 2006). Sollicitée en psychologie, en sociologie ou en socio-économie, la notion de confiance est-elle également pertinente en sciences de l'information, plus particulièrement pour la gestion des documents d'activités (records management) ${ }^{3}$, soit les documents produits dans l'accomplissement des processus métiers ? Pourrait-on également parler de la notion de confiance informationnelle et à l'aide de quels indicateurs pourrait-on l'opérationnaliser? Des éléments de réponse se trouvent dans la perception qu'ont les acteurs organisationnels des critères de qualité et de la valeur de l'information.

La confiance constitue un facteur susceptible d'influencer les comportements informationnels des acteurs organisationnels, par exemple lors de la recherche et l'utilisation d'information. La confiance peut aussi influencer les pratiques documentaires des acteurs organisationnels, c'est-à-dire la manière dont ils gèrent les documents créés dans la réalisation des processus métiers. On assiste souvent, dans les organisations, à une diversité de pratiques personnelles et collectives. Cela n'a rien d'étonnant si l'on se fie aux recherches portant sur la gestion personnelle de l'information (Barreau \& Nardi, 1995 ; Bellotti \&

\footnotetext{
1 Dominique Maurel est Professeure adjointe à l'École de bibliothéconomie et des sciences de l'information de l’Université de Montréal ; dominique.maurel@umontreal.ca

2 Aïda Chebbi est doctorante et assistante de recherche à l'École de bibliothéconomie et des sciences de l'information de l'Université de Montréal ; aida.chebbi@umontreal.ca

3 Dans une perspective intégrée, la Loi sur les archives du Québec (1983) définit les archives comme l'ensemble des documents, quelles que soient leur date ou leur nature, produits ou reçus par une organisation ou un individu dans l'exercice de ses activités, et conservés pour leurs valeurs administratives, financières, légales, et d'information générale. Les documents ont trois périodes d'activité : active, semi-active et inactive.
} 
Smith, 2000). Cet état de fait représente un risque à la constitution d'une mémoire organisationnelle consignée qui soit complète et fiable. Celle-ci constitue pourtant une ressource de gestion essentielle à la réalisation des processus métiers, de même qu'une trace informationnelle ayant valeur de preuve (Bergeron et al., 2009). Si les employés ne peuvent se fier à la mémoire consignée de leur organisation, cela influe négativement sur leur perception de la qualité de l'information disponible, tout comme sur leur perception de la fiabilité des pratiques documentaires formelles de leur organisation. La confiance informationnelle apparaît comme étant un corollaire essentiel de la gouvernance de l'information, celle-ci représentant un cadre de responsabilisation qui précise les normes relatives à la création, l'évaluation, l'utilisation, la conservation, l'accès et la sécurité de l'information pour permettre aux organisations d'atteindre leurs objectifs à travers une utilisation efficace et efficiente de l'information (McManus, 2004 ; Logan, 2010).

Nous proposons une définition opérationnelle du concept de confiance informationnelle. Les indicateurs proposés pour l'examiner reposent sur les critères de qualité et la valeur de l'information. Nous illustrons nos propos avec des données provenant d'une étude en milieu municipal. Notre objectif consiste à comprendre la dynamique entre la perception de la confiance informationnelle, les comportements informationnels et les pratiques documentaires privilégiées par des cadres intermédiaires de deux arrondissements d'une municipalité canadienne. Cette dynamique est complexe puisqu'elle inclut tant des dimensions cognitives, affectives que contextuelles, à des niveaux interpersonnel, organisationnel et institutionnel de la confiance informationnelle.

\section{Revue de littérature}

La notion de confiance informationnelle apparaît comme étant un élément important des comportements informationnels et des pratiques documentaires des acteurs organisationnels. Défini comme la somme des activités grâce auxquelles l'information devient utile pour un utilisateur, le comportement informationnel se décline en trois phases que sont l'identification des besoins d'information, la recherche d'information et l'utilisation d'information (Taylor, 1991 ; Wilson, 1997, 2000). Choo (2006) souligne les dimensions cognitives, affectives et contextuelles (ou situationnelles) des comportements informationnels.

Comportement informationnel et pratique documentaire (ou informationnelle) sont parfois utilisés de manière interchangeable (Savolainen, 2007 ; Chaudiron \& Ihadjadene, 2010). Toutefois, derrière le concept de pratique, prévaut l'idée d'une action récurrente et régulière dont les contours sont en partie déterminés par le contexte social et culturel environnant; de manière absolue, la pratique n'existe pas si les membres d'une communauté ne s'y engagent pas. Cela va dans le même sens que les pratiques partagées par 
un groupe de travailleurs exerçant la même profession, ou par les employés d'une même organisation. Ainsi, la praxis de l'information peut inclure toutes les actions documentaires liées à la création, l'évaluation, l'organisation, le repérage, la conservation et le transfert de l'information, dont le succès constitue une valeur ajoutée à l'utilisation de l'information dans un contexte social (organisationnel, par exemple) qui influe sur ces pratiques (Taylor, 1986 ; Savolainen, 2007).

\section{La confiance}

La confiance peut être définie comme "la croyance qu'un autre individu, une organisation ou une institution agira de façon conforme à ce qui est attendu de lui » (Simon, 2007, p. 85). Elle est caractérisée par une interdépendance et une réciprocité, tout comme par une certaine vulnérabilité entre les partenaires de la relation de confiance. Ceux-ci ont des attentes envers une relation de confiance honnête et coopérative, qui peut toutefois se voir entravée si l'un des partenaires fait preuve d'opportunisme et affaiblit ainsi les termes de la relation (Ford, 2001 ; Huotari \& Iivonen, 2004 ; Nooteboom, 2006 ; Delerue \& Bérard, 200 ; Laplante \& Harrisson, 2008). Les attentes ont une dimension sociale puisqu'elles s'appuient sur des valeurs partagées, par exemple au sein d'un même organisme ou d'une même profession (Ford, 2001). La confiance ne peut être forcée ou imposée : elle est volontaire, spontanée et naturelle; elle se co-construit dans les relations bilatérales qui s'établissent entre individus ou entre groupes (Long \& Sitkin, 2006). Les indicateurs de la confiance sont multiples puisqu'ils reposent sur des fondements affectifs, cognitifs et sociaux. Ils peuvent aussi s'appuyer sur des mécanismes organisationnels de contrôle tels que les normes ou procédures, ou sur des facteurs de contrôle social (ou institutionnels) comme le cadre juridique et réglementaire (Delerue \& Bérard, 2007).

Trois principaux types de confiance se dégagent des écrits sur le sujet (Thuderoz et al., 1999 ; Ford, 2001 ; Six, 2005 ; Sydow, 2006 ; Schoorman et al., 2007 ; Simon, 2007) :

- La confiance interpersonnelle prend place entre des individus, indépendamment de leur titre ou de leur poste. Cette confiance repose sur des bases affectives et cognitives.

- La confiance organisationnelle se produit quand les membres d'un organisme choisissent de s'engager dans une " action collective " (Simon, 2007 : 89) à titre de représentants de leur organisme respectif quand plus d'un organisme est en cause (confiance interorganisationnelle) ou de leur unité administrative dans l'organisme (confiance intraorganisationnelle).

- La confiance sociale ou institutionnelle est basée sur une structure sociale "formelle », soit le cadre juridique et réglementaire qui régit chaque société, ou encore le contexte normatif propre à une discipline. 
La confiance facilite la coopération et fait ainsi office de mode de coordination (Thuderoz et al., 1999 ; Simon, 2007). Dans les échanges intra et interorganisationnels, elle peut être considérée comme un instrument de gouvernance qui fait référence à des mécanismes de contrôle informel, lesquels peuvent se substituer en partie aux mécanismes de contrôle formel (Huotari \& Iivonen, 2004). Si la confiance organisationnelle n'est pas présente, il faut la construire en développant des normes, des obligations ou des valeurs partagées (Delerue \& Bérard, 2007).

\section{La confiance informationnelle}

Le partage d'information constitue l'un des facteurs de confiance entre individus ou groupes dans l'organisation. On pourrait, par extension, supposer qu'il existe une confiance informationnelle reposant notamment sur la perception des individus envers la qualité de l'information (et des sources d'information) et sa valeur (symbolique, d'affaires, de preuve, etc.). Différents critères de qualité de l'information sont relevés dans les écrits en gestion et en sciences de l'information.

Le critère de pertinence, en premier lieu, est déterminé par l'utilité de l'information lors de la résolution d'un problème, son exactitude, sa conformité aux faits et son actualité (ou " fraîcheur »). La fiabilité constitue un autre critère et repose sur la cohérence de l'information à travers le temps : des sources d'information fiables doivent être en mesure de maintenir un niveau acceptable d'exactitude dans le temps (Bergeron, 1996 ; Choo, 2002). Le critère de crédibilité est aussi proposé pour juger de la fiabilité d'une source, en fonction de la qualité et de l'exactitude de l'information qu'elle fournit (Wilson, 1997). L'authenticité des documents d'activités contribue à définir la qualité de l'information, critère essentiel lorsque les traces informationnelles ont valeur de preuve (Duranti, 1995).

Le critère de richesse des sources d'information réfère à la capacité de celles-ci à transmettre un maximum d'information pour comprendre la situation ou le problème à résoudre (Daft \& Lengel, 1984). Les sources d'information les plus riches et de plus haute qualité seraient, en ordre décroissant, les communications en personne, les communications téléphoniques et les communications écrites de nature personnelle ; autrement dit, les sources humaines et personnelles. Les sources d'information les moins riches et de moindre qualité seraient les communications écrites formelles, donc documentaires et impersonnelles.

L'accessibilité des sources d'information peut contribuer à accroître la perception de leur qualité, quoique les avis soient partagés à ce sujet. Certains affirment que l'accessibilité des sources représente un facteur plus important que leur qualité, et qu'une source plus accessible aura tendance à être utilisée même si sa qualité est moindre ; d'autres croient que la qualité prime sur l'accessibilité ; d'autres encore avancent que l'accessibilité des sources pourrait 
être un élément à considérer, mais seulement en relation avec leur crédibilité, leur fiabilité et leur objectivité (Wilson, 1997 ; Choo, 2002). Fidel \& Green (2004) constatent que le concept d'accessibilité recouvre aussi les dimensions de disponibilité et de facilité d'utilisation de l'information.

Feldman \& March (1981) suggèrent que l'utilisation de l'information s'inscrit dans certaines conventions sociales qui lui donnent un caractère symbolique. Ils relèvent trois caractéristiques qui font que l'utilisation de l'information, dans les organisations, ne se conforme pas au modèle rationnel de prise de décision : (1) un système d'incitatifs à recueillir plus d'information que nécessaire pour s'assurer d'avoir en main toute l'information utile au besoin ; (2) un système de surveillance de l'environnement qui favorise la collecte d'information ayant parfois peu de valeur immédiate dans une perspective décisionnelle, puisque la pertinence de l'information ne peut toujours être précisée à l'avance ; et (3) le fait que l'information peut avoir une valeur stratégique, s'avérer un instrument de pouvoir et être parfois volontairement déformée, par exemple dans un contexte de conflit d'intérêts. Une telle déformation stratégique de l'information amenuise la valeur et la fiabilité de celle-ci, ce qui peut entraîner les acteurs organisationnels à s'entourer de trop d'information. Feldman \& March (1981) proposent de considérer l'information à la fois comme symbole et signal : d'une part, comme un symbole puisqu'elle ne représente pas seulement un fondement qui se veut rationnel (celui du « choix intelligent ») à la décision, mais aussi la compétence et l'efficacité au sein de l'organisation ; l'utilisation de l'information permet notamment de légitimer les individus et les organisations qui prennent de «bonnes " décisions. D'autre part, l'information peut être vue comme un signal de l'« économie de l'information "si on arrive à démontrer que les organisations qui prennent de bonnes décisions sont en mesure de recueillir et de traiter l'information à un coût inférieur aux organisations qui prennent de moins bonnes décisions : il s'agit ici de savoir mesurer le prix de l'information, de sa collecte à son utilisation, et à ses conséquences sur la décision.

\section{Méthodologie}

Notre recherche est de nature descriptive et exploratoire, et s'inscrit dans une approche qualitative interprétative. Elle vise à explorer la manière dont les cadres intermédiaires répondent à leurs besoins d'information courante, et ce, en fonction de l'influence de dimensions individuelles (cognitives et affectives) et contextuelles sur les comportements informationnels. Les propos des répondants permettent d'identifier différents indicateurs de confiance, tant dans leurs comportements informationnels que dans leurs pratiques documentaires.

Les 21 répondants de notre étude sont des cadres intermédiaires provenant de deux arrondissements d'une municipalité québécoise fusionnée dans 
les années 2000. Le contexte d'une organisation municipale vivant des transformations majeures, suite aux fusions imposées par le gouvernement provincial québécois, et aux défusions subséquentes réclamées par certaines municipalités, représente un riche terrain d'étude. Le choix des cadres intermédiaires repose sur un échantillonnage à choix raisonné, de type non probabiliste, où les répondants présentent des caractéristiques typiques. L'homogénéité du groupe des cadres intermédiaires constitue un premier critère. Au plan de la structure hiérarchique, nous avons privilégié deux niveaux de responsabilités, en l'occurrence des chefs de division et des directeurs d'unités administratives. Les principaux modes de collecte de données ont été des entrevues en profondeur en personne avec les cadres intermédiaires et l'observation directe de ces derniers. Les données recueillies ont fait l'objet d'une analyse de contenu qualitative basée sur la théorisation ancrée.

\section{Présentation et discussion des résultats}

L'analyse des comportements informationnels et des pratiques documentaires s'est faite dans le contexte de situations de gestion propres aux cadres intermédiaires rencontrés. Ces derniers ont décrit deux situations de gestion chacun, pour un total de 42 situations dont 25 étaient nouvelles pour eux. Celles-ci portent sur le thème des ressources matérielles et immobilières (15 situations), des aspects juridiques, réglementaires et normatifs (11 situations), des ressources humaines (8 situations), des communications (4 situations) et des ressources financières (4 situations). Les situations nouvelles étaient considérées par les cadres comme étant complexes et présentant des risques (légaux, politiques ou économiques) en cas d'échec ; elles demandaient généralement des solutions sur mesure. Les cadres intermédiaires étant redevables de leurs actions à plusieurs instances au sein de la municipalité, ils ont exprimé l'importance d'analyser toute l'information pertinente qu'ils pouvaient se procurer. Ces situations nouvelles engendraient donc un état d'incertitude qui pouvait se résoudre en bonne partie en faisant appel à de l'information.

\section{Les comportements informationnels pour la recherche et l'utilisation d'information : les sources d'information et les critères de qualité}

Pour résoudre les situations de gestion dont ils étaient chargés, les cadres intermédiaires municipaux ont consulté un total de 187 sources d'information, composées de 102 sources humaines (55\%) et de 85 sources documentaires (45\%). S'ils ont utilisé davantage de sources humaines, la proportion de sources documentaires reste élevée. Les sources humaines internes consultées en plus grand nombre sont les collègues de la même unité administrative, d'autres unités dans le même arrondissement, et des services corporatifs de la ville centre. Les sources humaines externes sont constituées de personnes ressources d'autres organisations (municipalités, ministères, 
fournisseurs, etc.). Les résultats indiquent que les répondants ont consulté un nombre considérable de sources d'information internes ${ }^{4}$ à l'organisation, tant humaines que documentaires : 153 sources internes $(81,8 \%)$ contre 34 externes $(18,2 \%)$. Trois catégories de sources documentaires internes sont utilisées en priorité : documents de nature juridique et normative, dossiers des projets en cours, et documents de ressources humaines et financières. Pour les sources documentaires externes, ce sont surtout des documents de nature juridique et normative qui sont consultés. Ces résultats sont à mettre en relation avec les besoins informationnels manifestés par les répondants. Dans une proportion de $80 \%$, ces besoins portaient sur des éléments internes : philosophie de l'organisation, partage des pouvoirs et des compétences entre les arrondissements et les services corporatifs de la ville centre, fonctionnement interne de la ville après sa restructuration. Les besoins informationnels étaient aussi formés à $20 \%$ de facteurs externes : lois, règlements et directives du gouvernement provincial québécois, négociations avec des groupes de pression, etc. Le contexte organisationnel était fortement influencé par l'incertitude due aux réorganisations vécues par la municipalité, qui en subissait encore les contrecoups au moment de la collecte de données (Maurel \& Bergeron, 2010). Ce contexte explique en bonne partie le choix de sources d'information dignes de confiance.

Les critères de préférence des sources d'information humaines sont multiples. Ils sont tout d'abord en phase avec le rôle d'agent de liaison des cadres intermédiaires, alors que ceux-ci constituent et entretiennent des réseaux de relations professionnelles, tant à l'intérieur qu'à l'extérieur de l'organisation (Mintzberg, 1973 ; Kotter, 1982). Le rôle de liaison fait partie des rôles interpersonnels des cadres, ceux-ci entretenant des contacts formels et informels, horizontalement et verticalement dans la structure organisationnelle. Les personnes auprès de qui ils recueillent de l'information peuvent être impliquées à divers degrés dans la réalisation de leurs projets. Par exemple, un répondant doit compter sur les directeurs des autres directions pour obtenir les données exactes et complètes l'aidant à établir les prévisions budgétaires de l'arrondissement. En contrepartie, lorsque le budget demandé doit être réduit, chacun est amené à comprendre la réalité d'ensemble et les priorités de chaque direction, et à revoir ses prévisions budgétaires en conséquence (A-5).

Le fait que les cadres intermédiaires aient utilisé davantage de sources d'information humaines est à mettre en relation avec l'assertion de Choo (2002) voulant que les sources perçues comme fournissant l'information de la plus haute qualité soient le plus souvent personnelles et dignes de confiance.

4 Pour cette étude, nous associons les sources documentaires internes aux documents d'activités des organisations, créés et reçus par les employés dans le cadre des processus métiers. Les sources documentaires externes consistent essentiellement en de la documentation de référence. 
Les répondants ont très souvent recouru à des sources d'information humaines pour mieux comprendre les dimensions processuelles et contextuelles entourant une situation. Ainsi, la consultation de collègues a permis à un répondant d'obtenir l'historique (non consigné) d'une situation et de mieux saisir les tensions entre différentes parties: "J'avais des données, mais pas de comprébension du dossier. Les gens du comité ont été très gentils, il y en a un (...) qui siégeait avant, souvent il me donnait l'information " (A-4). La proximité et la facilité d'accès aux sources d'information représentent un critère important, au même titre que la pertinence et la fiabilité de l'information transmise par des collègues. Cela montre également l'utilisation que font les cadres intermédiaires de leur réseau de connaissances professionnelles.

Les cadres intermédiaires rencontrés ont néanmoins consulté une importante quantité de sources documentaires, ce qui soutient les propos de Feldman \& March (1981) sur la valeur symbolique de l'information : le fait d'appuyer les décisions sur de l'information permet de légitimer les individus qui prennent ces décisions, d'où le concept d'information comme symbole. Cela confirme aussi la volonté des individus de s'inscrire dans un processus de rationalité où les bonnes décisions doivent être fondées sur un processus de preuves 5 . Notre étude ne permet pas de vérifier si les cadres intermédiaires ont tendance à ne justifier qu'après coup, à l'aide de documents, les décisions prises. Les résultats indiquent toutefois qu'ils jugent essentiel d'appuyer leurs décisions sur des documents crédibles et fiables. Leurs propos démontrent qu'ils sont imputables envers différentes instances, par exemple leurs supérieurs immédiats, les membres des comités chargés d'approuver certains types de projets et de dépenses, les élus de leur arrondissement, etc.

De manière générale, les répondants ont comblé leurs besoins d'information de manière suffisamment satisfaisante pour résoudre les situations dont ils étaient chargés. Souvent, pour des raisons de temps, de disponibilité ou d'accessibilité à l'information, ils n'ont pas poursuivi leurs recherches de manière exhaustive. Certains ont fait comprendre qu'ils avaient recueilli assez d'information pour répondre à leurs besoins et ont arrêté la recherche. Dans les cas où ils n'ont pas trouvé de réponses claires, ils ont exercé leur jugement sur la base de leurs connaissances et de leur expérience. Ce comportement est aussi lié à la capacité d'absorption d'information des individus (Cohen \& Levinthal, 1990) quant à leur habileté à reconnaître la valeur d'une nouvelle information et à l'intégrer à leur bagage de connaissances. Enfin, cela est en lien avec le type de solution auquel tend l'individu, entre la recherche d'une solution satisfaisante (dans une perspective de rationalité limitée) ou la recherche de la meilleure solution parmi toutes celles qui existent (dans une

5 Pfeffer \& Sutton (2006) affirment l'importance du « evidence-based management ", où les gestionnaires ont avantage à s'appuyer sur des preuves constituées des sources d'information que possède déjà souvent l'organisation, de même que de l'examen critique de ces sources. 
perspective de rationalité absolue). En raison du temps dont ils disposaient, certains répondants ont dû faire des compromis et se sont concentrés sur les quelques sources d'information qui leur fournissaient des réponses satisfaisantes. Les propos des répondants permettent de croire que leurs besoins ont été comblés dès que l'information trouvée a été jugée suffisante pour formuler des réponses ou solutions adéquates. Dans un contexte de rationalité limitée (March, 1994), les critères de qualité de l'information semblent donc réduits au minimum requis.

Par ailleurs, le fait que les cadres intermédiaires aient utilisé plus de sources d'information internes qu'externes vient enrichir la discussion sur l'accessibilité physique des sources, notamment leur proximité. Ils se tournent avant tout vers les documents d'activités de leur organisation pour satisfaire leurs besoins d'information et fournir les justifications requises. Les collègues à l'interne constituent également un moyen, pour certains cadres intermédiaires, de repérer les sources documentaires pertinentes : «Si je trouve un document, c'est parce que j'ai trouvé le collègue » (B-28).

Le cadre de référence des cadres intermédiaires (formé de leurs rôles et responsabilités de gestion, de même que des facteurs contextuels immédiats) influe sur leur perception des situations à résoudre et, partant, sur leur perception des sources d'information adéquates qu'il serait essentiel de consulter. Le contexte constitue ainsi une variable importante dans la recherche de l'information nécessaire. Cela rejoint les propos de Dennis et Valacich (1999) à l'effet qu'il n'existe pas de richesse des sources d'information dans l'absolu.

\section{Le cas des systèmes d'information}

Les sources d'information consultées par les cadres intermédiaires sont souvent des systèmes d'information où sont consignés données et documents. Il s'agit essentiellement de : (1) systèmes formels externes à la municipalité $(8,25 \%)$ : sites Web de municipalités, ministères, fournisseurs, etc. ; (2) systèmes formels implantés dans la municipalité $(46,5 \%)$ : suivi des dossiers décisionnels, gestion comptable, suivi des requêtes des citoyens, messagerie électronique, Intranet, etc. ; (3) systèmes formels propres aux unités administratives des arrondissements ou des services corporatifs de la ville centre $(31,75 \%)$ : gestion comptable, suivi des projets, gestion de location d'équipements, serveurs communs en réseau partagés par des équipes de travail, etc. ; et (4) systèmes informels individuels conçus par les cadres intermédiaires ou leurs employés (13,5\%) : système comptable parallèle, veille technologique, suivi de projets, etc.

En matière d'indicateurs de confiance, les cadres intermédiaires ont exprimé des exigences pour les systèmes d'information utilisés. La qualité de l'information est essentielle et est exprimée en termes de crédibilité, fiabilité, exactitude et authenticité. Les droits d'accès et d'écriture aux 
personnes autorisées constituent une assurance en ce sens. L'adaptation aux particularités de recherche de chaque système d'information constitue un défi pour plusieurs cadres intermédiaires rencontrés : de manière générale, ils notent que les clés d'accès disponibles pour la recherche sont restreintes et s'appuient sur des données minimales; de plus, la nomenclature trop lourde, par exemple dans le cas de l'Intranet, ne leur permet pas de s'y retrouver facilement. Dans un tel cas, ils n'ont alors pas l'assurance de trouver toute l'information pertinente dont ils auraient besoin. Au point de vue de la performance générale des systèmes d'information, ceux-ci sont considérés peu fiables s'ils ne permettent pas d'établir les corrélations entre données souhaitées par les cadres, ou de produire des rapports suffisamment détaillés pour leurs besoins. Ces raisons sont généralement suffisantes pour que les cadres créent un système parallèle sur une application bureautique. Pour ce qui est de l'accessibilité à l'information, plusieurs répondants se doutent que de l'information pertinente est disponible dans d'autres systèmes pour lesquels ils ne disposent pas des droits d'accès. Pouvoir accéder à toute l'information disponible dans tous les systèmes de leur organisation leur permettrait, selon eux, de pouvoir regrouper l'ensemble des éléments d'information dont dispose chaque unité sur un même projet ; ainsi, le dossier du projet serait complet et, par conséquent, plus fiable et crédible.

Les systèmes d'information font aussi office d'outils de recherche pour les cadres intermédiaires, à l'aide des moteurs de recherche intégrés. Un critère de qualité essentiel pour les cadres est l'accès direct et rapide à l'information en format numérique depuis leurs ordinateurs personnels. Ils disent utiliser très peu de documents papier et, le cas échéant, font alors appel à leurs secrétaires qui les conservent dans leurs bureaux respectifs. L'accessibilité matérielle est donc un critère important, mais pas au détriment de la pertinence et de la validité de l'information. Les cadres intermédiaires doivent pouvoir justifier leurs décisions à plusieurs instances, sur la base de sources documentaires fiables, crédibles et authentiques.

Les sources documentaires sur lesquelles les cadres intermédiaires appuient leurs décisions, principalement internes à l'organisation, recèlent plusieurs valeurs. En premier lieu, la valeur d'affaires ou de preuve des décisions prises, des actions réalisées et de l'évolution des processus. Les cadres sont redevables à plusieurs instances et doivent ainsi justifier leurs décisions, particulièrement lorsque celles-ci sont, à leur tour, présentées et défendues par leurs supérieurs aux membres du conseil municipal ou aux citoyens. La valeur de preuve doit s'accompagner de l'assurance de l'authenticité des documents. Enfin, le fait de constituer systématiquement des dossiers complets documentant les décisions et conservés pour référence future relève de la valeur symbolique de l'information. 


\section{Les pratiques documentaires et les critères de qualité de l'information}

Les cadres intermédiaires ont tous des pratiques documentaires précises pour l'organisation de leurs documents d'activités. Leurs propos permettent de distinguer ce qui relève de pratiques organisationnelles et personnelles, particulièrement pour la classification et la conservation des documents (Maurel, 2010). Pour les pratiques documentaires de niveau organisationnel, la situation est quelque peu différente selon que les répondants appartiennent à un arrondissement qui était une municipalité à part entière avant la fusion municipale, ou à un arrondissement créé après la fusion. Les répondants de l'arrondissement ex-ville connaissent l'existence du système de classification uniforme en vigueur dans leur arrondissement et savent qu'il est maintenu par le records manager. Certains disent appliquer ce système de classification pour leurs documents papier. D'autres savent que ce système est implanté dans les secrétariats. Les répondants connaissent aussi l'existence de délais de conservation pour les documents qu'ils produisent, même s'ils n'en connaissent pas la durée exacte. Par ailleurs, certains ont mentionné que les documents à valeur permanente, et ceux ayant une valeur légale, sont conservés aux archives. Enfin, tant pour la classification que la conservation des documents, certains répondants ont référé aux actions posées par l'unité de records management. Celle-ci est reconnue comme détenant les compétences permettant d'assurer une saine gestion des documents d'activités. Il est donc intéressant de constater que les procédures organisationnelles de gestion des documents d'activités ne soient pas toujours respectées par les répondants.

La majorité des répondants ont des pratiques documentaires personnelles. Certains, par exemple, ont créé leur propre système de classement de documents sur support papier ou numérique. Les cadres intermédiaires de l'arrondissement créé lors de la fusion (et sans tradition de gestion des documents d'activités au moment des entrevues) ont exprimé des besoins pour un système uniforme de classement des documents d'activités. Ils conservent les dossiers des projets dont ils ont la responsabilité mais, dans certains cas, ont dit ne pas savoir s'ils conserveraient ces dossiers à long terme : cela dépendrait de leurs besoins et de l'espace de rangement disponible. Généralement, les dossiers dont les cadres intermédiaires ont besoin de manière immédiate se trouvent sur leur table de travail, rangés par piles. Lorsque les situations sont résolues, les documents papier sont rangés dans les classeurs de leur bureau ou du secrétariat ; les documents numériques sont déposés sur le disque dur de leur ordinateur ou de systèmes d'information. Plusieurs documents sont en libre partage entre les membres de l'équipe sur un serveur réseau commun. Les dossiers conservés dans les bureaux contiennent souvent des commentaires personnels qui enrichissent la compréhension des situations, mais qui sont éliminés par la suite. Quelques répondants ont aussi fait part de l'importance de protéger l'information de nature confidentielle et légale. 
Par ailleurs, certaines pratiques documentaires se trouvent en quelque sorte déterminées par la nomenclature et les fonctionnalités des systèmes d'information utilisés. Les systèmes d'information étant endossés par l'organisation qui en a fait l'acquisition, on peut considérer que les pratiques documentaires qu'ils entraînent s'apparentent à des pratiques de niveau organisationnel. Dans certains cas, les caractéristiques fonctionnelles des systèmes semblent contrevenir aux durées de conservation déterminées par l'unité de records management. Un répondant décrit ainsi le cas d'un système dédié au traitement de réclamation des citoyens dans son arrondissement : "Tout ce qui est là-dedans c'est ineffaçable, c'est impossible de les effacer.»(A-6). Cette situation suggère qu'un des systèmes d'information les plus importants développés par les records managers (le calendrier de conservation des documents) n'est plus jugé nécessaire pour déterminer la durée de vie des documents. Les capacités de stockage ont amené les concepteurs du système de traitement des réclamations à outrepasser complètement cette question au profit d'une approche centrée sur la conservation permanente des traces associées au processus. Le répondant explique d'ailleurs que la majorité des informations archivées ne sont jamais utilisées, mais qu'elles agissent comme police d'assurance pour la ville en cas de problèmes éventuels. Par ailleurs, pour ce répondant, la confiance envers l'unité de records management n'est pas acquise pour ce qui est de l'accessibilité des documents d'activités : "Je sais que des fois, l'archiviste, ça semble un peu le fatiguer de voir qu'il y a tant de documents en double et en triple. Nous, on lui explique que ça nous les prend. Je ne peux pas prendre pour acquis que c'est quelque part dans un autre département, et que le jour où je vais en avoir besoin, je vais appeler quelqu'un pour m'envoyer une copie» $(A-6)$. Cet exemple met en lumière la dynamique du pouvoir dans le développement et la mise en place des pratiques documentaires dans cette organisation. Cet exemple n'est pas unique. Plusieurs des cadres intermédiaires rencontrés ont développé un ou des systèmes parallèles pour la réalisation de leurs activités de gestion, ce qui entraîne la création de documents numériques stockés sur ces seuls systèmes et qui risquent d'échapper en partie aux pratiques documentaires décidées pour l'ensemble de l'organisation.

La personnalisation des pratiques documentaires est bien ancrée, et semble rassurer les répondants quant à l'accessibilité immédiate, la disponibilité en tout temps et la fiabilité de l'information. Pour les pratiques documentaires de niveau organisationnel, les cadres intermédiaires comptent sur leur organisation pour préserver la version finale, "officielle ", authentique, ayant valeur de preuve. Ces pratiques sont institutionnalisées au sens où elles sont entérinées par l'unité de records management et par les instances de l'organisation. Elles répondent aux exigences légales de niveau provincial (Québec) et fédéral (Canada) en matière de gestion des documents, d'accès aux documents, de protection des renseignements personnels et de droits 
d'auteur. L'unité de records management pourrait aussi se prévaloir des normes internationales existantes (par exemple, les normes ISO en information et documentation). Ce cadre juridique et normatif donne une crédibilité aux pratiques de gestion des documents d'activités de l'organisation.

\section{Types de confiance, comportements informationnels et pratiques documentaires}

Les résultats obtenus permettent aussi une réflexion sur les trois types de confiance que sont la confiance interpersonnelle, organisationnelle et institutionnelle. On observe des manifestations des trois types de confiance tant dans les comportements informationnels (recherche et utilisation de sources d'information) que dans l'adoption de pratiques de gestion des documents d'activités.

En ce qui a trait à la confiance interpersonnelle, sans surprise, les sources humaines sont les plus utilisées. Il s'agit tout probablement d'un réflexe naturel, en raison de la facilité d'obtention des renseignements contextualisés et nuancés. Les sources sont alors constituées de personnes dans l'entourage professionnel immédiat des cadres intermédiaires, à qui ils font confiance car elles ont prouvé leur capacité à fournir de l'information fiable et pertinente dans leur champ de compétences. Les critères de qualité, ici, semblent donc être principalement l'accessibilité, la fiabilité, la pertinence et la crédibilité de l'information (celle-ci étant basée sur la crédibilité de la personne consultée).

Pour ce qui est des manifestations de la confiance organisationnelle ou plutôt, ici, intra-organisationnelle, on remarque que les sources d'information humaines sont essentiellement des personnes que les cadres intermédiaires ne connaissent pas toujours personnellement, mais à qui ils accordent leur confiance en tant que représentantes de leur unité. Par le fait même, ces personnes devraient détenir un niveau d'expertise suffisant pour que l'information donnée soit fiable et crédible. Il existe un risque de déséquilibre si les partenaires de la relation de confiance manipulent ou retiennent des éléments d'information à leur avantage. Certains répondants ont mentionné des disparités entre le discours officiel de l'organisation et les actions posées dans les unités en période de restructuration, ce qui a eu comme effet de créer un sentiment d'insécurité. Les critères de qualité observés sont ici la fiabilité, la pertinence et la crédibilité de l'information diffusée en regard des gestes concrets posés par la haute administration.

Dans une perspective de records management, il faut en arriver à développer la confiance des employés envers les dispositifs de gestion des documents d'activités mis en place. Des éléments de confiance possibles sont les outils normatifs comme les procédures, le système de classification des documents d'activités, les moteurs de recherche ou les index facilitant le repérage. Il importe de s'assurer que les documents d'activités sont gérés efficacement de manière à pouvoir être réutilisés au moment adéquat par les membres 
de l'organisation. Un autre élément de confiance est lié aux systèmes d'information organisationnels soutenant la réalisation des processus métiers ; cette confiance se manifeste par des critères de performance (corrélation de données, production de rapports, etc.), de facilité et de rapidité de recherche, ainsi que de rigueur dans la gestion des droits d'écriture (ce qui est en lien avec l'intégrité et l'authenticité de l'information). Ces critères supposent une collaboration entre les unités des technologies de l'information et de records management, et avec les utilisateurs qui sont aussi les spécialistes de contenus. Ainsi pourra se développer une confiance accrue de la part des utilisateurs.

Enfin, la confiance sociale ou institutionnelle se manifeste à travers la structure légale et normative. Cette confiance s'appuie notamment, en ce qui a trait aux documents d'activités des organisations, sur des éléments dont l'autorité est reconnue, par exemple les lois et normes professionnelles servant de fondement aux actions de l'unité records management. De ces lois et normes découle le programme de gestion des documents d'activités, avec ses directives et procédures adaptées à l'organisation. On pourrait donc s'attendre à ce que cette structure légale et normative crée un sentiment d'assurance chez les employés envers l'unité records management, et envers les pratiques documentaires formelles recommandées. Thuderoz et al. (1999, p. 52) affirment que "ce sont les institutions qui permettent aux autres modes de confiance d'exister". Il est légitime de croire qu'un cadre juridique, réglementaire et normatif constitue une bonne base à la confiance informationnelle dans les organisations, considérant la valeur de preuve des documents d'activités à conserver, les mesures entourant la protection de l'information confidentielle, etc. On peut toutefois se demander si la confiance institutionnelle est vraiment suffisante pour susciter les deux autres types de confiance.

Pour reprendre les termes de la définition de la confiance donnée par Simon (2007), il faut examiner si les dispositifs institutionnels et organisationnels de la confiance informationnelle, tels qu'ils sont mis en œuvre, font agir les membres de l'organisation de façon conforme à ce que l'on attend d'eux. Ces dispositifs sont-ils suffisants pour susciter la confiance de manière à modifier les comportements informationnels et les pratiques documentaires? Il faudrait que les records managers puissent créer un sentiment d'interdépendance et de réciprocité dans la relation de confiance informationnelle qui s'établit entre eux et les membres de l'organisation qui sont leurs " clients ». Si les acteurs organisationnels ont des attentes envers les records managers pour la gestion des documents d'activités, les records managers ont également des attentes envers eux : ils s'attendent en effet, minimalement, à ce que les procédures mises en place soient respectées par tous les employés. Ainsi pourra se construire une mémoire organisationnelle consignée qui soit 
complète et fiable. La gouvernance de celle-ci en assurera non seulement la préservation, mais la réutilisation stratégique pour des situations de gestion similaires. Actuellement, avec la tension existant entre pratiques documentaires personnelles et collectives (ou organisationnelles), il semble que la gouvernance de l'information au sein des deux arrondissements soit plutôt une « gouvernance négociée " dans son application quotidienne. Mais cette négociation n'en est pas vraiment une. Les acteurs organisationnels créent des solutions documentaires qui leur sont propres et qui correspondent à leurs besoins, sans en avoir discuté avec le records manager auparavant. Il se manifeste ici une dynamique de pouvoir en l'absence de véritables éléments de négociation, d'autant plus que la confiance devrait normalement être un élément important de toute négociation (Thuderoz et al., 1999).

\section{Conclusion}

Cet article a permis une réflexion sur la notion de confiance et, par extension, sur la notion de confiance informationnelle. Les indicateurs de la confiance informationnelle (notamment les critères de qualité et de valeur symbolique de l'information) peuvent s'observer tout d'abord dans les comportements informationnels des cadres intermédiaires étudiés. Aux étapes de la recherche et de l'utilisation d'information, les types de sources consultées et les raisons sous-jacentes à ces choix permettent de bien comprendre les exigences des cadres face à l'information, aux sources et aux systèmes d'information. Les indicateurs de la confiance informationnelle peuvent aussi s'observer dans les pratiques documentaires des cadres intermédiaires, alors que s'exerce une tension entre des éléments de pratique personnelle et organisationnelle. Il en résulte une gouvernance négociée de l'information au quotidien, ou plutôt une absence de négociation et un déséquilibre dans l'effort de gouvernance.

Il faudrait maintenant recueillir davantage de données empiriques sur les manifestations de la confiance informationnelle des acteurs organisationnels. Des études supplémentaires permettraient, dans un premier temps, de mieux définir et documenter les indicateurs de la confiance informationnelle. Elles permettraient, dans un deuxième temps, de développer une compréhension plus exhaustive des relations entre ces indicateurs et les trois types de confiance (interpersonnelle, organisationnelle et institutionnelle). Cet article a abordé la question sous l'angle des documents d'activités issus des processus métiers. Il serait pertinent d'observer l'opérationnalisation de la notion de confiance en lien avec la gestion des connaissances et la veille stratégique, afin d'analyser comment se négocie la gouvernance informationnelle dans ces contextes spécifiques. 


\section{BIBLIOGRAPHIE}

BARREAU D.K., NARDI B.A., "Finding and reminding: file organization from the desktop », SIGCHI Bulletin, 27, 3, 1995, p. 39-43.

BELLOTTI V., SMITH I., "Informing the design of an information management system with iterative fieldwork ", Proceedings of the $3^{\text {rd }}$ Conference on Designing Interactive Systems, 2000, p. 227-237.

BERGERON P., "Information resources management ", Annual Review of Information Science and Technology, 31, 1996, p. 263-300.

BERGERON P. et al., "La gestion stratégique de l'information », in SALAÜN J.-M. et ARSENAULT C., Introduction aux sciences de l'information, Montréal, QC, Presses de l’Université de Montréal, 2009, p. 183-205.

CHAUDIRON S., IHADJADENE M., « De la recherche de l'information aux pratiques informationnelles », Études de communication, 35, 2010, p. 13-30.

CHOO C.W., Information management for the intelligent organization: the art of scanning the environment, Medford, NJ, Information Today, 2002.

$\mathrm{CHOO} \mathrm{C} . \mathrm{W}$., The knowing organization: how organizations use information to construct meaning, create knowledge, and make decisions, New York, NY, Oxford University Press, 2006.

COHEN W.M., LEVINTHAL D.A., "Absorptive capacity: a new perspective on learning and innovation ", Administrative Science Quarterly, 35, 1, 1990, p. 128-152.

DAFT R.L., LENGEL R.H., " Information richness: a new approach to managerial behavior and organization design ", Research in Organizational Behavior, 6, 1984, p. 191-233.

DELERUE H., BERARD C., « Les dynamiques de la confiance dans les relations interorganisationnelles », Revue Française de Gestion, 33, 175, 2007, p. 125-138.

DENNIS A.R. et VALACICH J.S., « Rethinking media richness: towards a theory of media synchronicity ", Proceedings of the 32nd Hawaii International Conference on System Sciences, January 5-8, 1999, Maui, Hawaii, 1, 1999, 10 p.

DURANTI L., " Reliability and authenticity: the concepts and their implications ", Archivaria, 39, 1995, p. 5-10.

FELDMAN M.S., MARCH J.G., " Information in organizations as signal and symbol », Administrative Science Quarterly, 26, 2, 1981, p. 171-186.

FIDEL R., GREEN M., "The many faces of accessibility: engineers' perception of information sources ", Information Processing and Management, 40, 2004, p. 563-581.

FORD D., Trust and knowledge management : the seeds of success, Working paper WP 01-08, Queen's KBE Centre for Knowledge-Based Enterprises, Kingston, ON, Queen's School of Business, 2001.

HUOTARI M.-L., IIVONEN M., " Managing knowledge-based organizations through trust ", in HUOTARI M.-L., IIVONEN M. (éd.), Trust in knowledge management and systems in organizations, Hershey, PA, Idea Group Publishing, 2004, p.1-29.

KOTTER J.P., The general managers, New York, NY, The Free Press, 1982. 
LAPLANTE N., HARRISSON D., " Les conditions de la confiance entre gestionnaires et représentants syndicaux dans un contexte d'innovations ", Relations Industrielles, 63, 1, 2008, p. 185-104.

LOGAN D., "What is information governance? And why is it so hard? ", 2010, http://blogs.gartner.com/debra_logan/2010/01/11/what-is-information-governanceand-why-is-it-so-hard/

LONG C. P., SITKIN S.B., "Trust in the balance : how managers integrate trust-building and task control ", in BACHMANN R., ZAHEER A. (éd.), Handbook of trust research, Northampton, MA, Edward Elgar, 2006, p. 87-103.

MARCH J.G., A primer on decision making : how decisions happen, New York, NY, The Free Press, 1994.

MAUREL D., «Systèmes d'information organisationnels et réalisation des processus d'affaires : où interviennent les processus documentaires? ", Actes du $38^{e}$ Congrès annuel de l'Association canadienne des sciences de l'information, Montréal (Québec), 2-4 juin 2010, 2010.

MAUREL D., BERGERON P., "Linformation au cœur des rôles de gestion ", Archives, 41, 2, 2010, p. 115-134.

MCMANUS J., "Working towards an information governance strategy », Management Services Journal, 48, 8, 2004, p. 8-13.

MINTZBERG H., The nature of managerial work, New York, NY, Harper and Row, 1973.

NOOTEBOOM B., "Forms, sources and process of trust ", in BACHMANN R., ZAHEER A. (éd.), Handbook of trust research, Northampton, MA, Edward Elgar, 2006, p. 247-263.

PFEFFER J., SUTTON R.I., « Evidence-based management », Harvard Business Review, January, 2006, p. 63-74.

Québec (province), Loi sur les archives, Chapitre A-21.1, 1983.

SAVOLAINEN R., "Information behavior and information practice: reviewing the "umbrella concepts" of information-seeking studies ", The Library Quarterly, 77, 2, 2007, p. 109-132.

SCHOORMAN F.D., MAYER R.C., DAVIS J.H., "An integrative model of organizational trust: past, present, and future ", Academic of Management Review, 32 2, 2007, p. 344-354.

SIMON É., "La confiance dans tous ses états », Revue Française de Gestion, 33, 175, 2007, p. 83-94.

SIX F., The trouble with trust: the dynamics of interpersonal trust building, Northampton, MA, Edward Elgar, 2005.

SYDOW J., "How can systems trust systems? A structuration perspective on trust-building in inter-organizational relations ", in BACHMANN R., ZAHEER A. (éd.), Handbook of trust research, Northampton, MA, Edward Elgar, 2006, p. 377-391.

TAYLOR R.S., Value-added process in information systems, Norwood, NJ, Ablex, 1986.

TAYLOR R.S., "Information use environments ", Progress in Communication Sciences, 10, 1991, p. 217-255. 


\section{$\mathrm{C} \& O \mathrm{n}^{\circ} 42$}

THUDEROZ C., MANGEMATIN V., HARRISSON D., La confiance : approches économiques et sociologiques, Boucherville, QC, Gaëtan Morin Éditeur, 1999.

VALLEE P.-H., MACKAAY E., " La confiance : sa nature et son rôle dans le commerce électronique ", Lex Electronica, 11, 2, 2006, p. 45.

WILSON T.D., " Information behaviour: an interdisciplinary perspective ", Information Processing and Management, 33, 4, 1997, p. 551-572.

WILSON T.D., "Human Information Behavior ", Informing Science, 3, 2, 2000, p. $49-56$.

Résumé : En nous appuyant sur les écrits portant sur la confiance, nous proposons la notion de confiance informationnelle. Les critères de qualité et de valeur de l'information pourraient permettre d'en observer les manifestations en contexte organisationnel. La confiance peut influencer les comportements informationnels en matière de recherche et d'utilisation d'information, tout comme les pratiques documentaires utilisées pour gérer les documents d'activités dans le cadre des processus métiers. Nous illustrons ces propositions avec les résultats d'une étude de comportements informationnels portant sur des cadres intermédiaires d'une municipalité canadienne. Nous cherchons à comprendre la dynamique entre la perception de la confiance informationnelle, les comportements informationnels et les pratiques documentaires privilégiées par ces cadres, de même que les résultats de cette dynamique sur la gouvernance de l'information dans l'organisation. Mots-clés : Confiance, qualité de l'information, valeur de l'information, comportement informationnel, pratique documentaire, document d'activité, processus métier.

Abstract : Building on trust literature, we propose the concept of informational trust. Criteria such as quality and value of information could make it possible to observe the demonstrations of trust in organizations. Trust can influence information behaviours as well as documentary practices used to manage records generated through business processes. We illustrate these assumptions with the results of a study of middle managers' information behaviours in a Canadian municipality. Our objective is to study the dynamics taking place between the perception of informational trust, the information behaviours and the documentary practices favored by middle managers, and the results of this dynamics on information governance in organizations.

Keywords : Trust, quality of information, Value of information, Information behaviour, Documentary practice, Record, Business process. 